\title{
sUrbane Wachen und die neuen Polizeien. Wenn im Quartier die Randständigen ins Zentrum rücken
}

\section{VOLKER EICK}

Bis Anfang der 1990er Jahre durfte als gesichert gelten, dass in der Bundesrepublik die Bewohner >abgehängter Stadtquartiere sicherheitsund ordnungspolitisch Gegenstand der Landespolizeien, sozialpolitisch der Sozial- und Arbeitsämter sowie sonstiger (lokal)staatlicher Behörden waren. Das hat sich mit dem Bedeutungsgewinn des Lokalen gründlich geändert. Denn diese Akteure wurden ergänzt um (und herausgefordert von) private(n) Selbsthilfeinitiativen und neue(n) soziale(n) Bewegungen, die in den 1980er Jahren zunächst in Opposition zum Lokalstaat auftraten. Unter Begriffen wie Nonprofit- oder Dritt-Sektor-Organisationen in den (kritischen) Sozialwissenschaften kategorisiert (vgl. Anheier et al. 1999), wurden sie nach und nach in den Behördenapparat eingebunden und machten gleichzeitig einen Institutionalisierungs- und Professionalisierungsprozess durch (vgl. Eick et al. 2004: 56ff.).

Zum anderen gewann ein weiterer privater Akteur an Bedeutung, das kommerzielle Sicherheitsgewerbe, das ebenfalls mit Beginn der 1990er Jahre seinen Einfluss auf die Kontrolle des öffentlichen Raums ausweitete und seine Bedeutung in zahlreichen Kooperationsvereinbarungen mit Landes- und Bundespolizeien sowie kommunalen Ordnungsämtern schriftlich fixieren und so vergrößern konnte (Stober/Olschok 2004: 571ff.). Sehr zu Recht aber fragte Detlef Nogala vor einigen Jahren, was "eigentlich so privat an der Privatisierung sozialer Kontrolle« sei und kritisierte, dass »oftmals Phänomene autonomen oder staatsbeaufsichtigten Vigilantismus' mit denen der kommerziellen zivilen Sicherheitsindustrie vermengt« würden (Nogala 1995: 258). Er betont damit die un- 
terschiedlichen Handlungslogiken von Bürgerwehren (autonome zivilgesellschaftliche Organisationsform), Freiwilligen Polizeidiensten und Bürgerwachten (staatlich beaufsichtigte Organisationsform) sowie von Sicherheitsdiensten (profitorientierte Organisationsform). Michael Lindenberg (2000) hat mit dem Begriff »kommunale Hilfsdienste« herausgestellt, dass mit Instrumenten der aktiv(ierend)en Beschäftigungspolitik Erwerbslose und Sozialhilfeempfangende im Auftrag der Kommunen Sauberkeits-, Ordnungs- und/oder Sicherheitsdienstleistungen (im Folgenden: SOS-Dienstleistungen) erbringen. Beide Politikfelder werden so verknüpft. Seit rund 15 Jahren gewinnen diese surbanen Wachen in bundesdeutschen Städten an Bedeutung. Auf der lokalen Ebene hat sich eine neue Ordnungsarbeit (die Produktion von Sauberkeit, Ordnung und Sicherheit im öffentlichen Raum) entwickelt, die neuen Akteuren der Sicherheitsproduktion und neuen Konkurrenz- und Kooperationsbeziehungen zum Durchbruch verholfen hat. Entsprechend ist von einer "pluralization of policing « von der »transformation « oder »multilateralization of policing«, von einer neuen »culture of control« die Rede. Es wird das Aufkommen von »third-party«, "private«, "para« und »nonprofit policing« konstatiert, von der »militarization of policing « ist die Rede, und es werden neue Formen des »hybrid« und »nodal policing« identifiziert. Schließlich ist von der »extended policing family « in einer »mixed economy of policing « die Rede. ${ }^{1}$ Mein Ansatz versucht, diese Beobachtungen zu präzisieren und insbesondere die Verknüpfung von Beschäftigungs- und Sicherheitspolitik als (lokales) Ordnen im Wandel zu konzeptionalisieren. Ich werde zunächst einige theoretische Anmerkungen zur Einordnung machen und sodann zeigen, dass sich auch in anderen westeuropäischen Ländern Programme identifizieren lassen, die lokale Sicherheits- und Beschäftigungspolitik miteinander verknüpfen. Schließlich wird anhand des Bund-Länder-Programms >Soziale Stadt` gezeigt, dass mit solchen Programmen ein Bedeutungs- und Einflussgewinn für Nonprofit-Organisationen bei der Problemdefinition und -bearbeitung auf der lokalen Ebene verbunden ist.

1 Aus Platzgründen verzichte ich auf die bibliographischen Angaben der hier zitierten Begriffe; sie gehen zurück auf folgende Autoren: David Bealey, Clifford Shearing, Michael Kempa, Trevor Jones, Tim Newburn, David Garland, Michael Buerger, Lorraine Mazerolle, Philip Stenning, George Rigakos, Volker Eick, Peter Kraska, Les Johnston, Adam Crawford, Stuart Lister, Nigel South. 
Tabelle 1: Varianten des Schumpeterian Workfare Post-National Regime

\begin{tabular}{|ll}
\hline Neoliberalismus \\
\hline 1. & Liberalisierung - Stärkung des freien Wettbewerbs \\
2. & Deregulierung - Zurückdrängen der Rolle von Recht und Staat \\
3. & Privatisierung - (Aus-)Verkauf der öffentlichen Infrastruktur \\
4. & Verbreitung der Marktlogik im verbliebenen öffentlichen Sektor \\
5. & Internationalisierung - »freier « Handel nach Innen und Außen \\
6. & Senkung direkter Steuern - Stärkung der Nachfrage(auswahlmög-lichkeiten) \\
\hline
\end{tabular}

\section{Neostaatlichkeit}

1. Von der Staatskontrolle zur regulierten Konkurrenz

2. begleitende nationalstaatliche Strategie statt top-down-Politik

3. Beaufsichtigung des privaten und öffentlichen Sektors

4. Public Private Partnerships (PPP) unter staatlicher Führung

5. Neomerkantilistischer Schutz der Kernökonomie

6. Ausweitung der Rolle »neuer« kollektiver Akteure

\section{Neokorporatismus}

1. Neuausrichtung von Konkurrenz und Kooperation

2. Dezentralisierte »regulierte Selbstregulation«

3. Ausweitung und Ausdifferenzierung privater, staatlicher und anderer "stakeholder"

4. Ausweitung der Rolle von PPP

5. Schutz der Kernökonomie in einer offenen Ökonomie

6. hohe Besteuerung zur Finanzierung sozialer Infrastruktur (to finance social investment)

\section{Neokommunitarismus}

1. Deliberalisierung - Begrenzung des freien Wettbewerb

2. Empowerment - Erweiterung der Rolle des Dritten Sektors

3. Sozialisierung - Ausweitung der sozialen Ökonomie

4. Betonung des sozialen Gebrauchswerts und sozialen Zusammenhalts

5. fairer, nicht freier Handel; think global, act local

6. Umlenkung von Steuern - Bürgerlohn (citizens' wage, carers' allowances)

Quelle: nach Jessop 2002.

Ich gehe davon aus, dass wir gegenwärtig einen Prozess - ausgehend von den 1980er Jahren in den USA (Harvey 2005) - beobachten, der mit dem Begriff neoliberale Globalisierung beschrieben werden kann. Neoliberale Globalisierung wird hier in regulationstheoretischer Perspektive analysiert, also als Suche nach einem neuen Akkumulationsmodell und einer kohärenten Regulationsweise nach der Krise des Fordismus (vgl. 
Demirovič et al. 1992; Brand/Raza 2003). Jessop (2002) fasst Neoliberalismus als Liberalisierung, Privatisierung und Deregulierung, wobei sich Liberalisierung auf den Grad der Marktöffnung bezieht. Privatisierung repräsentiert die Veränderung der Eigentumsverhältnisse in vormaligen staatlichen Unternehmen bzw. Institutionen. Deregulierung beschreibt die im Zuge von Liberalisierung und Privatisierung notwendigen Maßnahmen zur Schaffung und Erhaltung eines funktionierenden Wettbewerbs und der Absicherung infrastruktureller Erfordernisse.

Diese Neoliberalisierung ordne ich in den parallel zu beobachtenden, analytisch aber davon zu trennenden, Prozess der Globalisierung ein, die treffender mit dem Begriff Glokalisierung zu bezeichnen ist (Swyngedouw 1997). Wo und wie sich jeweils stärker neoliberale, neostaatliche, neokorporatistische bzw. neokommunitaristische Elemente herausbilden, ist dabei von kulturellen und sozialen Traditionen, konkreten ökonomischen Rahmenbedingungen etc. abhängig und ein in sich widersprüchlicher und ungleichzeitig verlaufender Prozess. Auf der lokalen Ebene zeichnet sich ab, dass neokorporatistische und neokommunitaristische Elemente zur Stabilisierung und Flankierung des neoliberalen Projekts zum Einsatz kommen.

Brenner und Theodore (2002) fassen den aktuell existierenden Neoliberalismus dialektisch als einen Prozess, der einerseits auf Zerstörung, andererseits auf der Entwicklung neuer Regulationsmodi und Institutionen basiert. Neoliberale Zerstörung kann danach als Beseitigung keynesianischer Artefakte (öffentlicher Wohnungsbau, öffentlicher Raum etc.), entsprechender Politiken (umverteilende Wohlfahrtsstaatlichkeit), Institutionen (öffentliche Wohnungsbaugesellschaften, Gewerkschaften etc.) und Vereinbarungen (fordistischer Arbeitskompromiss, Umverteilung von Geldern der Bundes- auf Länder- und Städteebene etc.) beschrieben werden. Demgegenüber kann die kreative Seite des Neoliberalismus in der Etablierung neuer oder der Neuausrichtung bestehender Institutionen und Praxen gesehen werden, die der (zukünftigen) Reproduktion des Neoliberalismus dienen (Konsortien unter Beteiligung von Regierung und Staat, Public Private Partnerships; Workfare-Politiken etc.).

Jamie Peck und Adam Tickell (2002) haben analog und in historischer Perspektive vorgeschlagen, die Etablierung des Neoliberalismus nach einer proto-, roll-back- und roll-out-Phase zu unterscheiden. Handelte es sich in der ersten Phase im wesentlichen um ein ideologisches Projekt, getragen von einem feinmaschigen Netz von Think tanks (Peck/Tickell 2007), werden in der zweiten Phase keynesianische Politiken und Artefakte (zunächst) zerstört, um Platz für die dritte Phase zu schaffen - ein roll-out von stärker proaktiven neoliberalen Praxen und Elementen auf allen räumlichen Ebenen und in allen gesellschaftlichen 
Kontexten. Während dabei der Nationalstaat an Bedeutung verliere, gewännen sowohl die supranationale wie die lokalstaatliche Ebene an Bedeutung. Wie erwähnt, schlägt Swyngedouw (1997) für diese Prozesse neoliberaler Globalisierung vor, den Begriff Glocalization zu verwenden, weil so die gleichzeitige Verlagerung von nationalstaatlichen Regulationsaufgaben snach oben ( auf die globale Ebene und ihre Institutionen) und snach unten Robertson 1998). Der Rückgang nationalstaatlicher Interventionen - etwa bei Wohnungsversorgung, lokaler Infrastruktur, Wohlfahrt etc. zwingt dabei die lokalstaatliche Ebene, solche Interventionen selbst wahrzunehmen und zu finanzieren, sie in Kooperation mit der privaten Wirtschaft zu erbringen oder sich aus ihnen zurückzuziehen - und sie so den `Kräften des Marktes` zu überlassen.

\section{Lokale Sicherheits - und Beschäftigungspolitik durch Staat, Markt und Nonprofits}

Für den Bereich der Inneren Sicherheit und die Beschäftigungspolitik heißt das zunächst, dass eine ganze Palette neuer Akteure mit dem Erbringen von SOS-Dienstleistungen beauftragt ist (vgl. Tabelle 2), wobei der Begriff >zivilgesellschaftlich « hier mit Gramsci (1991ff.) benutzt werden soll.

Tabelle 2: Ausgewähle staatliche und nicht-staatliche Sicherheitsakteure

\begin{tabular}{|l|l|l|}
\hline \multicolumn{1}{|c|}{ staatlich } & zivilgesellschaftlich & \multicolumn{1}{c|}{ kommerziell } \\
\hline Polizeien der Länder & $\begin{array}{l}\text { Nonprofit- } \\
\text { Organisationen }\end{array}$ & Sicherheitsdienste \\
\hline Bundespolizei (Ex-BGS) & Bürgerwachten & Detekteien \\
\hline Bundesfinanzpolizei (Zoll) & Bürgerwehren & Personenschützer \\
\hline Ordnungsämter & $\begin{array}{l}\text { Neighborhood } \\
\text { Watch }\end{array}$ & Türsteher \\
\hline Sicherheitswachten & Sicherheitspartner & Werkschutz \\
\hline Ordnungspartnerschaften & $\begin{array}{l}\text { Freiwilliger Polizei- } \\
\text { dienst }\end{array}$ & (Söldner) \\
\hline
\end{tabular}

Quelle: Eigene Darstellung 
Mit Joachim Hirsch kann Zivilgesellschaft zunächst als heterogenes Netzwerk von vom Staat formell mehr oder minder unabhängigen Organisationen gefasst werden, die der

»Formulierung und Vertretung gesellschaftlicher Interessen, der politischen Willensbildung, der Meinungsäußerung und der öffentlichen Diskussion dienen. $[\ldots]$ Bei genauerer Betrachtung handelt es sich [...] um von der Struktur der kapitalistischen Gesellschaft bestimmte Bestandteile des institutionellen Regulationssystems, mittels dessen Herrschaft stabilisiert und der Akkumulationsprozess des Kapitals garantiert wird. Die Institutionen der zivilen Gesellschaft sind selbst von ökonomischen Macht- und politischen Herrschaftsverhältnissen geprägt« (Hirsch 2002: 59f.).

Daher geht der Bedeutungsgewinn der Zivilgesellschaft nicht mit dem Abnehmen von Zwang einher, sondern, eher im Gegenteil, mit seiner Zunahme. "Es ist freilich ein Zwang, der versucht, im Alltag so weit als möglich zu verschwinden« (Kebir 1991: 66). Zivilgesellschaft im gesellschaftlichen Mainstream-Diskurs fungiert jedoch häufig als klassischer >Container-Begriff`, in den gleichsam alles `Gute` gepackt werden kann (vgl. Breckner 1997; Mayer 2003; DeFilippis/Fisher/Shragge 2006). Zivilgesellschaft ist mithin auch in den Sozialwissenschaften sumkämpftes Terrain ‘. In diesem Sinne sind die seit den 1990er Jahren aufgebauten >zivilgesellschaftlichen< Organisationen (vgl. Tab. 2), die Landes- und Bundesprogramme aktivierender Beschäftigungs- und lokaler Stabilisierungspolitik $^{2}$ in dieser Ambivalenz zu fassen. Weitgehend einheitlich werden bei der Würdigung kommerzieller Sicherheitsdienste und kommunaler Kriminalprävention als neue Trends die kleinräumige Orientierung solcher Initiativen sowie eine als Privatisierung staatlicher Aufgaben charakterisierte Entwicklung hervorgehoben. ${ }^{3}$ Auch die Herausbildung von Operativen Gruppen und Sondereinheiten der Landespolizeien, des Bundesgrenzschutzes und des Zolls als Bundesfinanzpolizei, die sich gezielt spezifischer Bevölkerungsgruppen oder städtischer Räume annehmen, belegen diese Trends. Als Bedeutungsgewinn des Lokalen kann auch die (Re)Kommunalisierung durch den Aufbau von Außendiensten der Ordnungsämter gefasst werden (Beste 2001; Eick 2004); das kommerzielle Sicherheitsgewerbe hat seine Arbeitsfelder bei gleichzeitigem Wachstum ausdifferenziert. Um nur einen Überblick zu den

2 Das Bund-Länder-Programm >Soziale Stadt` und die analogen Landesprogramme zielen auf die soziale Stabilisierung der Quartiere und das Empowerment der Zivil- bzw. Bürgergesellschaft (vgl. Eick 2005).

$3 \mathrm{Zu}$ privaten Sicherheitsdiensten vgl. für viele: Mahlberg (1988); Nitz (2000); zur Kommunalen Kriminalprävention vgl. Kury (1997); Pütter (2006); zu Bürgerwehren vgl. Hitzler (1994). 
Tätigkeitsfeldern in alphabetischer Reihenfolge zu geben: Asylbewerberlager, Abschiebegefängnisse, City-Streifen, Facility Management, Fahndung, Gefängnismanagement, Kommunale Kriminalprävention, Maritime Sicherheit, Quartiersmanagement, Parkraumbewirtschaftung, Psychatrische Anstalten, Radarkontrollen, Security Points, Sicherheitsdienste im ÖPNV, Umweltschutz (`Ranger`), Verkehrsüberwachung, Videoüberwachung, Zweiter Arbeitsmarkt (Olschok 2004; Eick 2007b; eigene Erhebungen).

Bisher wenig Aufmerksamkeit wurde hingegen Hilfs- und Ordnungsdiensten gewidmet, die in der vergangenen Dekade unter der (An)Leitung freier gemeinnütziger Träger in diesem Marktsegment tätig geworden sind, weil sie, zumindest auf den ersten Blick, keine ordnungs- oder kriminalpolitischen Funktionen zu haben scheinen (vgl. Lindenberg 2000).

Auch die Arbeitsmarkt- und Sozialpolitik hat sich in den vergangenen Jahren stark verändert. Die aktive Arbeitsmarktpolitik, in der Bundesrepublik bisher klassisch auf so genannte Zielgruppen orientiert, verortet sich heute verstärkt sozialräumlich und fühlt sich lokalen Ansätzen verpflichtet. ${ }^{4}$ Modifizierungen und Neuerungen, die in den 1990er Jahren im Arbeitsförderungsrecht und Bundessozialhilfegesetz auf nationalstaatlicher Ebene vorgenommen wurden, sollten der lokalen Ebene mehr Handlungsspielräume eröffnen. Zudem sind unabhängig davon zahlreiche neue Kooperationsformen und Handlungsansätze in der Arbeitsmarkt- und Sozialhilfepolitik auf lokaler Ebene auch jenseits rechtlicher Vorgaben entstanden, in die zunehmend privatwirtschaftliche Anbieter einbezogen werden. Die Parallele zu den oben skizzierten Entwicklungen im Bereich Innerer Sicherheit überrascht wenig, folgt sie doch der gleichen Logik: Nur durch das gemeinschaftliche Handeln, so die These, können hinreichend Problemlösungskapazitäten zur Verfügung gestellt werden. Das entspricht dem Bedeutungszuwachs, den das Lokale und die Gemeinschaft bzw. der für die deutsche Diskussion weniger verfängliche Begriff der Community in den vergangenen Jahren gewonnen haben (Rose 1996; Lindenberg 2000). Auch die Stadtentwicklungs- und Wirtschafts(förderungs)politik orientieren sich zunehmend an lokalen Strukturen im aktivierenden Sozialstaat, den Bob Jessop (2002) mit dem Begriff des »Schumpeterian Workfare Post-National Regime« und Joachim Hirsch (2002) mit dem Begriff des »nationalen Wertbewerbsstaats $«$ fassen.

4 Eick et al. (2004); vgl. für die Soziale Arbeit: Kessl/Otto (2004); für sozialstaatliche Veränderungen insgesamt: Dahme et al. (2003). 
Paradigmatisch für derartige Handlungsansätze sind die seit Mitte der 1990er Jahre in verschiedenen Bundesländern entwickelten Quartiersmanagementkonzepte und seit 1999 das Bund-Länder-Programm >Soziale Stadt`(Becker/Löhr 2000; Walther 2002). Integrierte, ressortübergreifende und ganzheitliche Ansätze sollen in sbenachteiligten Wohngebieten einen Beitrag zur Verbesserung der dortigen Lebensbedingungen leisten und die Bevölkerung zur aktiven Mitarbeit animiert werden. Solche Ansätze sind mit einer Vielzahl von `Zumutungen` ausgerechnet für diejenigen verbunden, die ohnehin besonders von den neoliberalen Verwüstungen betroffen sind und mit Arbeitslosigkeit, Armut und Krankheit zu kämpfen haben.

Programmatisch zielt das Bund-Länder-Programm auf die soziale Stabilisierung >benachteiligter« Quartiere und ihrer BewohnerInnen, die mit Empowerment zu eben dieser Stabilisierung raktiviert len. Schließlich geht es um eine Verwaltungsmodernisierung, die ressortübergreifend und (fiskalische) Ressourcen schonend in den lokalstaatlichen Administrationen eine betriebswirtschaftliche Handlungslogik entwickeln und durchsetzen soll (vgl. im Überblick Dahme et al. 2003). Mit diesem als (sozial)integrativ bezeichneten und auf die (Wieder)Herstellung sozialer Kohäsion zielenden Ansatz scheint das Quartiersmanagement anknüpfungsfähig sowohl an die Gemeinwesenarbeit der 1960er oder 1970er Jahre, an (Vorläufer)Programme wie die behutsame Stadterneuerung der 1980er Jahre und an die in den 1990er Jahren intensiv geführte Debatte um das Neue Steuerungsmodell in den Verwaltungen. ${ }^{5}$ Es fehlt eine einheitlich akzeptierte Definition für Quartiersoder Stadtteilmanagement, ${ }^{6}$ aber es destillierten sich rund 20 Handlungsfelder für das Quartiersmanagement heraus, die sich vor Ort in diversen Zielkatalogen niederschlagen (vgl. Tabelle 3).

$\mathrm{Zu}$ Beginn dominierten investiv-bauliche Maßnahmen das Programm. Instandsetzungen, teilweise Neubauten und umfassende Wohnumfeldmaßnahmen waren kennzeichnend. Während hier noch weitgehend einheitliche Vorgehensweisen identifiziert werden können, lassen sich in anderen Handlungsfeldern große Differenzen von Fall zu Fall und von Ort zu Ort identifizieren. Damit sei eine besondere Qualität des Programms verbunden, argumentieren einige Autoren, weil so eine zielgenaue und problemadäquate Reaktion auf lokale Probleme wahrschein-

5 Grimm (2004: 135) schreibt, »[f]ür die Stadtplanung knüpft Quartiermanagement an die Strategie der sbehutsamen Stadterneuerung an [...], die Gemeinwesenarbeit sieht darin eine Weiterentwicklung des Arbeitsansatzes [...], und im Einzelhandel werden damit Strategien bezeichnet, die trading-down-Prozessen [...] entgegenwirken sollen « (Hervorh. im Orig.). Vgl. Alisch (1998); Hinte (1998); Walther (2002); Grimm (2004: 130ff.). 
licher werde (Franke/Löhr 2000; Grimm 2004: 133f.). Während sie dabei meist hoffnungsfroh auf das Programm und seine zivilgesellschaftlichen Potentiale blicken, lassen sich vielfältige (neue) Ausgrenzungsprozesse identifizieren. Im Handlungsfeld >Tolerantes Zusammenleben « finanziert ein Berliner Quartiersmanagement einerseits Sprachkurse für Migranten, die staatlicherseits keinen Anspruch auf solche Förderung haben (so in Kreuzberg), in einem anderen Quartiersmanagement gibt es andererseits Zuzugsbeschränkungen für arabische und türkische Großfamilien (so in Neukölln)

\section{Tabelle 3: Handlungsfelder in der sozialen Stadt}

\begin{tabular}{|c|c|c|}
\hline \multicolumn{3}{|c|}{$\begin{array}{l}\text { Deutschland } \\
\text { Bund-Länder-Programm } \\
\text { ¿Stadtteile mit besonderem Entwicklungsbedarf - die soziale Stadt< }\end{array}$} \\
\hline $\begin{array}{l}\text { Strategische } \\
\text { Handlungsfelder }\end{array}$ & \multicolumn{2}{|c|}{$\begin{array}{l}\text { Inhaltliche } \\
\text { Handlungsfelder }\end{array}$} \\
\hline Ressourcenbündelung & Beschäftigung & Stadtteilkultur \\
\hline $\begin{array}{l}\text { Integrierte } \\
\text { Handlungskonzepte }\end{array}$ & $\begin{array}{l}\text { Qualifizierung } \\
\text { und Ausbildung }\end{array}$ & $\begin{array}{l}\text { Sport } \\
\text { und Freizeit }\end{array}$ \\
\hline Quartiersmanagement & $\begin{array}{l}\text { Wertschöpfung } \\
\text { im Gebiet }\end{array}$ & $\begin{array}{l}\text { Zusammenleben im } \\
\text { Stadtteil }\end{array}$ \\
\hline $\begin{array}{l}\text { Aktivierung } \\
\text { und Beteiligung }\end{array}$ & $\begin{array}{l}\text { Soziale Aktivitäten und } \\
\text { soziale Infrastruktur }\end{array}$ & $\begin{array}{l}\text { Wohnungsmarkt und } \\
\text { Wohnungsbewirtschaftung }\end{array}$ \\
\hline Evaluation & Schulen und Bildung & $\begin{array}{l}\text { Wohnumfeld und öffentli- } \\
\text { cher Raum }\end{array}$ \\
\hline & Gesundheitsförderung & $\begin{array}{l}\text { Image und } \\
\text { Öffentlichkeitsarbeit }\end{array}$ \\
\hline & $\begin{array}{l}\text { Umwelt } \\
\text { und Verkehr }\end{array}$ & $\begin{array}{l}\text { Befähigung, Artikulation, } \\
\text { politische Partizipation }\end{array}$ \\
\hline \multicolumn{3}{|l|}{$\begin{array}{l}\text { Berlin } \\
\text { Quartiersmanagement }\end{array}$} \\
\hline & $\begin{array}{l}\text { Mehr Chancen auf dem } \\
\text { Arbeitsmarkt }\end{array}$ & $\begin{array}{l}\text { Besseres } \\
\text { Gesundheitsniveau }\end{array}$ \\
\hline & $\begin{array}{l}\text { Mehr Fort- und } \\
\text { Weiterbildung }\end{array}$ & $\begin{array}{l}\text { Besseres } \\
\text { Sicherheitsniveau }\end{array}$ \\
\hline & $\begin{array}{l}\text { Verbesserungen des } \\
\text { Wohn- und Lebens- } \\
\text { raumes }\end{array}$ & $\begin{array}{l}\text { Tolerantes } \\
\text { Zusammenleben }\end{array}$ \\
\hline & $\begin{array}{l}\text { Bewohneradäquate } \\
\text { soziale Infrastruktur }\end{array}$ & $\begin{array}{l}\text { Mehr Partizipation } \\
\text { der Bewohner/Akteure }\end{array}$ \\
\hline & $\begin{array}{l}\text { Verbesserte Stadtteil- } \\
\text { kultur }\end{array}$ & \\
\hline
\end{tabular}

Quelle nach: Eick 2007b, m.w.N. 
Zudem hat das Programm explizit nicht das Ziel, Arbeitslosigkeit, Armut oder soziale und ökonomische Ausgrenzung zu bekämpfen (HäuBermann/Kapphan 2000: 263). Aus dieser Perspektive sind die lokalstaatlichen Aktivitäten, zu denen das Outsourcing von Tätigkeitsfeldern aus den Verwaltungen, die Einschaltung von Nonprofit-Organisationen und (intermediären) privatwirtschaftlichen Agenturen sowie das Empowerment gehören, als Flankierung des neoliberalen Projekts interpretierbar. Gleichzeitig wird so auch klar, dass der lokale Staat und seine Intermediären nicht etwa nur Getriebene, sondern auch treibende Akteure bei der aktivierenden Ausgestaltung neuer Governance-Strukturen auf der lokalstaatlichen Ebene sind.

Ein Blick in die Niederlande und nach Großbritannien zeigt, dass die Verknüpfung von Beschäftigungsprogrammen mit Sicherheitspolitik, die Integration von Nonprofit-Organisationen und die Fokussierung auf den lokalen Raum keine deutschen Eigenheiten sind. ${ }^{7}$ Was auf den ersten Blick wie eine Perfidie spezifisch deutscher Vorstellungen von sozialer Integrationspolitik auf der lokalen Ebene aussieht, ist vielmehr gängige Praxis auch in anderen westeuropäischen Ländern.

\section{Ein Blick nach Westeuropa}

Am eloquentesten (und elaboriertesten) darf sicher das britische Modell gelten, das mit dem New Deal for Communities (NDC), einem von sieben New Deals, die New Labour unter Tony Blair einführte, und den Anti-social Behaviour Orders (ASBOs) ein Gemisch aus hoch moralisierenden Ordnungsvorstellungen und repressiven Elementen im Rahmen seiner neokommunitaristisch ausgerichteten »extended policing family« aufgebaut hat. ${ }^{8}$ Innerstädtische Konsumzonen und Wohnquartiere des Sozialen Wohnungsbaus werden mit quasi-polizeilichen Kräften überzogen, CCTV-Systeme ${ }^{9}$ haben sich allgegenwärtig durchgesetzt. Auch in den Niederlanden, noch bis vor wenigen Jahren nahezu der Inbegriff einer offenen und toleranten Gesellschaft, hat sich mit den Stadswachten $^{10}$ und Politiesurveillants ein vergleichbarer Politikwechsel vollzogen. Sie wurden zur Unterstützung und Entlastung professioneller Poli-

7 Die Ausführungen des nachfolgenden Unterkapitels basieren auf Eick (2007b).

8 Vgl. Levitas (1998/2005); Burney (2005); Flint (2006).

9 CCTV - closed circuit television, vernetzte Videoüberwachung.

10 Stadswachten stellen zudem den seltenen Fall eines Policy transfers von Kontinentaleuropa nach Großbritannien dar, denn dort wurde das Modell mit den Neighborhood Wardens kopiert. 
zeikräfte seit 1989 in zahlreichen niederländischen Gemeinden eingerichtet, um im öffentlichen Raum als Sondereinheiten, als Beschäftigungsprojekte oder als freiwillige Polizeieinheiten zu agieren. Generell sind deren Angehörige mit Uniformen und Funkgeräten ausgerüstet, haben keine polizeilichen Vollmachten, sind unbewaffnet und sollen durch Präsenz im öffentlichen Stadtraum das Sicherheitsgefühl der Bevölkerung anheben.

Neben einem Verkehrskontrolldienst (VICs, Veilgheid, Informatie, Controle - Sicherheit, Information, Kontrolle), der explizit als Beschäftigungsprojekt etabliert wurde, hat sich in den vergangenen Jahren das System urbaner Wachen in den Niederlanden in sozialräumlicher Hinsicht weiter ausdifferenziert: Stadtwachten patrouillieren in den innerstädtischen Gebieten zur Abschreckung gegen Unordnung und (Klein)Kriminalität, zeigen uniformierte Präsenz und dienen durch Sprechfunkverbindung und Tätigkeitsprotokolle als verlängerter Arm der Polizei; Gebietswachten haben spezifische Areale im innerstädtischen Raum zu kontrollieren wie etwa Busstationen oder Parks, sind aber auch als Verkehrspolizei etwa bei Baustellen oder Umleitungen im Einsatz; Präventionswachten schließlich kümmern sich um Opfer von Verbrechen und sind in der Kriminalprävention etwa mit Hinweisen zum besseren Schutz von Eigentum tätig; Letztgenannte sind auf Anfrage auch dazu da, während der Ferienzeit leere Wohnungen im Auge zu behalten.

Ähnlich wie in Kanada (Rigakos 2002) können Bürger, Einzelhandelsverbände oder Nonprofit-Organisationen zusätzliche staatliche Polizeikräfte `kaufen`, d.h. gegen Entgelt in ihren Nachbarschaften oder Geschäftsstraßen einsetzen - ein für Deutschland gegenwärtig noch schwer vorstellbares Verfahren (vgl. aber Oschmann 2005: 321ff.). Das unter Tony Blair eingeführte, auf >benachteiligte` Quartiere zielende Bundesprogramm NDC finanziert diese Polizeiarbeit (Crawford/Lister 2004: 22ff.). Weiter kommen so genannte Police Special Constables zum Einsatz. Waren sie zunächst vor allem als ergänzende Reserve in Fällen von inneren Unruhen und Notfällen gedacht, besteht ihre Rolle seit den späten 1960er Jahren darin, als einfache Bürger die Polizei zu unterstützen. ${ }^{11}$ Schließlich sind als dritte Variante lokaler Sicherheitsproduktion nicht-vereidigte Polizisten - Police Community Support Officers (PCSOs) - mit limitierten Rechten im Einsatz. Sie werden nach Security PCSOs, Community PCSOs und Transport PCSOs unterschieden, die

11 Rund 16.000 Police Special Constables - »a big unrecognized army« stellen gegenwärtig rund $13 \%$ der insgesamt 120.000 regulären Polizeikräfte (Gaston/Alexander 2001: 60). 
jeweils eigene Aufgabenbereiche haben. ${ }^{12}$ Im Jahr 2005 gab es allein in England und Wales 4.000 PCSOs; ihre Zahl soll nach Regierungsplänen bis zum Jahr 2008 um weitere 20.000 Personen aufgestockt werden (Johnston 2005: 243). Auch für deren Finanzierung wird auf Bundesprogramme wie das NDC oder das Programm Neighbourhood Renewal Funding (NRF) zurückgegriffen. Weiter haben seit Mitte der 1990er Jahre die Bezirke begonnen, eigene Gemeindepolizeien aufzustellen; sie arbeiten vor allem in den von der Gemeinde verwalteten Sozialwohnungsgebieten und lassen sich nach Crawford und Lister (2004: 28ff.) nach Local Authority Patrols und Neighbourhood Warden Schemes unterscheiden.

In Schottland, seit 1999 durch ein entsprechendes Memorandum der Bevölkerung mit mehr eigenständigen Rechten gegenüber der britischen Zentralregierung ausgestattet und dadurch in die Lage versetzt, selbst lokale Politiken umsetzen zu können, operieren seit 1996 City Centre Representatives (CCR) als urbane Wachen, die ebenfalls explizit als Beschäftigungsprogramm mit SOS-Anteilen eingerichtet worden sind. Das CCR-Programm wird auf dem Zweiten Arbeitsmarkt aus dem städtischen Haushalt, dem NDC-Programm und aus dem Europäischen Sozialfonds (ESF) finanziert und durch lokale Nonprofit-Organisationen umgesetzt. Die Reps, wie die City Centre Representatives umgangssprachlich in Glasgow genannt werden, bieten Informationen zu Verkehrsverbindungen, touristischen Sehenswürdigkeiten etc. Sie haben auch die Aufgabe, auf Sauberkeit und Ordnung in der Stadt zu achten und gelten daher, verbunden mit einem telefonischen Informationssystem zu ihrer Zentrale, als >Augen und Ohren « der Polizei. Ihre Funktion geht damit über ein bloßes Informationssystem für Touristen und Einheimische weit hinaus. Auf ihren Patrouillengängen besuchen sie die Geschäfte in den Hauptgeschäftsstraßen, nehmen umfassende Protokolle zu Auffälligkeiten auf (Unrat auf den Straßen, defekte Stadtmöbel etc.) und leiten diese an die zuständigen Behörden weiter (Helms 2003).

Anders als etwa in Trikont-Staaten (vgl. Trotha 2005; Eick 2006; Schulze in diesem Band), geht es bei deren Einsatz nicht um Staatszer-

12 Während Community PCSOs auf Patrouillengängen in festgelegten Gebieten sichtbar Präsenz zeigen sollen, sind Transport PCSOs im öffentlichen Nahverkehr in ganz London eingesetzt; Security PCSOs sind gegründet worden »for the purpose of carrying out anti-terrorist patrols « (Johnston 2005: 243). Der - angeblich missverstandene - Vorschlag von Bundesverkehrsminister Wolfgang Tiefensee, Hartz IV-Empfänger als »Volkssturm gegen El Kaida« einzusetzen (Leo 2006: 1), geht aber, soweit ersichtlich, nicht auf dieses Vorbild zurück. 
fall oder -versagen, also um einen zu schwachen Nationalstaat. ${ }^{13} \mathrm{Zu}$ beobachten ist in den entwickelten westlichen Industriestaaten vielmehr der neoliberale Umbau des Nationalstaats auf der Suche nach einem neuen kohärenten Wachstumsregime. Er greift dafür auf das spezifische Wissen und die spezifischen Fähigkeiten von supra- (EU, IWF, Weltbank etc.) und para-staatlichen Akteuren (G8, G10 etc.) ebenso zurück, wie zivilgesellschaftliche und lokalstaatliche Akteure in diesen Umbauprozess involviert werden.

\section{Workfare im Quartier}

Das betrifft beispielsweise den Bereich aktiver Arbeitsmarktpolitik sowie die Veränderungen der Beschäftigungspolitik insgesamt, die auf den Abbau von Leistungsrechten, verstärkte Sanktionen bei fehlender Kooperation(sbereitschaft), die (zwangsweise) Vermittlung in Niedriglöhne und die Gewährung von Leistungen nur noch bei erbrachten Gegenleistungen (Workfare) fokussieren (vgl. Eick et al. 2004). Kommerzielle Anbieter, das Quartiersmanagement und beschäftigungspolitisch aktive Nonprofits greifen in dieser Situation - noch vor Hartz IV und Agenda 2010 - zu punitiven und repressiven Maßnahmen. Helga Spindler (2003:

12) nennt sie treffend »Agenturen für Arbeitsdienste«, u.a. auch deshalb, weil sie »dem Arbeitsamt gegenüber Hinweise auf Arbeitsunwilligkeit von Maßnahmeteilnehmern« geben (Eick 2003a: 370) und in >benachteiligten`Quartieren Leistungsempfänger zum Teil deutlich unter dem Tarif- bzw. ortsüblichen Lohn beschäftigen (Eick et al. 2004: 144ff.). Dem Quartiersmanagement am Kottbusser Tor (Kreuzberg) gilt es offenbar bei der Ausbildungsplatzvermittlung von Jugendlichen als Erfolg, wenn durch die Auflagen "rund die Hälfte der Anfragenden abgeschreckt « wird (zit.n. Fritsche 2003: 86): »Wesentliche Funktion der Arbeitsplatzvermittlung ist die Lebensstilregulierung, die durch die Konfrontation der Jugendlichen mit Arbeitsmarkttugenden wie Pünktlichkeit, Sauberkeit und Zuverlässigkeit erreicht werden soll« (ebd.: 87). Eine Vertreterin aus einem der mittlerweile neun Quartiersmanagementgebiete im Berliner Bezirk Neukölln berichtete auf einer Veranstaltung der Friedrich-Ebert-Stiftung, dass »wir dafür gesorgt haben, dass ein Jugendhilfeträger keine Förderung durch das Arbeitsamt mehr erhält, weil der sich

13 Zwar sind etwa im Bundesland Brandenburg so genannte Sicherheitspartnerschaften installiert worden, um dem Aufkeimen von Bürgerwehren entgegenzuwirken (Eick 2000; Hohmeyer 2000), nur hat das mit Staatsverfall nichts zu tun. 
geweigert hat, mit der Polizei zusammenzuarbeiten. $«{ }^{14}$ Diejenigen Nonprofit-Organisationen, die in der aktivierenden Beschäftigungspolitik tätig sind und sich zu regelrechten Oligopolisten in den Quartieren entwickelt haben, ${ }^{15}$ setzen die neuen Vorgaben nach Hartz IV und Agenda 2010 zunehmend über Ein-Euro-Jobs um (Eick 2007b). Auch wenn die Kontrollen durch Landesregierungen und Kommunen eng und die Berichtspflichten nahezu kafkaesk sind - 14tägig müssen Arbeitsberichte gefertigt werden -, haben die QM-Teams vor Ort einen weiten Spielraum, ihre Vorstellungen von Quartiersentwicklung, von Sauberkeit, Ordnung und Sicherheit, durchzusetzen.

\section{Kriminalprävention im Quartier}

So sind die Quartiersmanager denn auch vielfältig in die Produktion von Sicherheit und Ordnung eingebunden. Damit ist an sich kein neues Spannungsfeld etwa zwischen Sozialarbeit und Polizei entstanden, denn Konflikte zwischen diesen beiden Akteursgruppen bestehen spätestens seit den 1970er Jahren. Das Bestreben der Polizei in die (StraBen)Sozialarbeit eingebunden zu werden bzw. ihren ordnungspolitischen Blick der Jugendarbeit zu oktroyieren, ist vielfach belegt (vgl. Gangway 1997: 291ff.), und unlängst hat Norbert Pütter (2006) das Interesse der Polizei an buchstäblich allen verfügbaren Informationen aus kriminalpräventiven Gremien dargelegt. Zudem handelt es sich beim Personal des Quartiersmanagement nicht mehr nur um Sozialarbeiter, sondern vielmehr um Stadtplaner, Politologen oder Betriebswirte, die in diesen intermediären Organisationen tätig sind und die Zugangsbedingungen für die Polizei in die Quartiersstrukturen erweitert und erleichtert haben. In einem Neuköllner Quartiersmanagement etwa meldete das Quartiersteam der polizeilichen >Operativen Gruppe Jugendgewalt`(OGJ) diejenigen (Migranten)Jugendlichen namentlich und mit Adressen, die ihnen als Intensivtäter galten. Die Jugendlichen erfuhren entsprechend eine spezielle Behandlung durch die Polizei und sind einem rigiden Kontrollregime - ohne vorherige Einschaltung von Sozialarbeit - unterworfen.

14 Die Tagung fand am 11. Mai 2004 unter dem Titel `Ethnische Kolonien, Ghettos und Parallelgesellschaften $<$ in Berlin statt.

15 Das kann aus Platzgründen hier nicht entwickelt werden; gemeint ist damit, dass einige Nonprofits in sihren< Quartieren den Großteil von aktiven Beschäftigungsmaßnahmen und mithin auch die Gelder der Arbeitsagenturen erfolgreich und nahezu ausschließlich auf sich (Oligopol) vereinigen können. 
Sozialpolitische Problemlagen werden so in einen ordnungspolitischen Kontext gestellt und Sozialarbeit zurückgefahren, die sich zudem zunehmend kriminalpolitisch erklären und rechtfertigen muss. Pütter (2002: 78) hat darauf hingewiesen, dass durch die Beliebigkeit des Präventionsbegriffes (nahezu) alle »lokal sicht- oder erfahrbare[n] Phänomene in eine kriminalpräventive Argumentation eingebunden werden « können ${ }^{16}$ und - unter Ausklammerung parlamentarischer Verfahren - selektiven Beteiligungsmechanismen und einer Entpolitisierung sozialpolitischer Problemlagen Vorschub geleistet wird. Einige Autoren betonen zudem, dass die Installation solcher Programme mit dem Abbau sozialstaatlicher Leistungen einherging und -geht. Entsprechend charakterisierten sie Kriminalpräventive Räte als »unbefriedigende Versuche, die Demontage des Sozialstaats zu kompensieren« (zitn. n. Eick 2005: 182). Kriminalprävention ist mittlerweile gleichsam natürlicher Bestandteil des Bund-Länder-Programms, und mit teilweise bedrückendem Halbwissen wird dem Broken Windows-Ansatz ${ }^{17}$ das Wort geredet ${ }^{18}$ und damit einem punitiven Paternalismus der Weg bereitet.

Da beiden Programmen - Quartiersmanagement und Kommunaler Kriminalprävention - die Vorstellung gemein ist, alle Akteure hätten an einem Strang zu ziehen und im Sinne einer (Selbst)Aktivierung zusammenzuarbeiten, sind sie explizit (Präventionsräte) oder implizit (QM) Einfallstor für ordnungs- und sicherheitspolitisch begründete Handlungsstrategien. Gleichzeitig stellen sie eine Zugangs- und Wissensressource der Polizei dar.

\section{Ordnung im Quartier}

Doch auch ohne explizite Integration von Ordnungsämtern oder Polizeikräften in das Quartiersmanagement spielen Sauberkeit, Ordnung und Sicherheit dort eine zunehmend wichtige Rolle. Dabei ist es einigermaßen schwierig, die entsprechenden Aktivitäten zu identifizieren, denn sie firmieren meist schlicht als Beschäftigungsmaßnahmen (mit oder ohne Qualifizierungsanteil), die nach dem SGB II, SGB III, aus EU- oder Sonderprogrammen finanziert werden, ohne dass deutlich gemacht wird,

16 Pütter (2002: 78) schreibt erläuternd, »der Jugendtreff wird nicht eingerichtet, weil die Jugendlichen einen eigenen Ort brauchen, sondern damit sie nicht mehr die Anwohner öffentlicher Plätze belästigen [...], Randgruppen werden aus den Innenstädten nicht wegen städtischer Imageinteressen verdrängt, sondern zur Sicherung des Sicherheitsgefühls etc.«

17 Vgl. dazu Dreher/Feltes (1997); McArdle/Erzen (2001).

18 Vgl. Schuleri-Hartje (2002), Kürpick/Murböck (1997). 
mit welchen Aufgaben Erwerbslose und Sozialhilfeempfangende in den Maßnahmen konkret beauftragt sind.

Einige Beispiele aus den insgesamt 33 Berliner QM-Gebieten (2007) können jedoch erhellen, worin die Tätigkeiten solcher Dienste bestehen und warum es gerechtfertigt scheint, hier von neuen Ausgrenzungsagenturen im aktivierenden lokalen Sicherheits- und Ordnungsstaat zu sprechen. Begünstigt durch die Skandalisierung linker Aktivisten vor Ort, die mit öffentlichkeitswirksamen Aktionen der Ausgrenzung so genannter Randgruppen (Wohnungslose, Trinkermilieus, Migrantenjugendliche) entgegentraten, sind die Aktivitäten von Quartiersmanagement und Nonprofit-Sicherheitsdiensten am Helmholtzplatz im Berliner Bezirk Prenzlauer Berg mittlerweile gut dokumentiert. ${ }^{19}$ Der Helmholtzplatz steht, wiewohl durch wissenschaftliche Studien im Vorfeld des Quartiersmanagement als >Verdachtsgebiet` für sozialen Niedergang ausgewiesen, unter Gentrifizierungsdruck (Senatsverwaltung für Stadtentwicklung 1998). Zugleich ist er aber auch beliebter Treffpunkt für die lokalen und Teile des überlokalen Trinkermilieus, Punks, Obdachlose, Trebekids und einige Konsumenten sowie Händler illegal(isiert)er Drogen (gewesen). Eine neu hinzugezogene kaufkraftstarke und konsumfähige Mittelschicht mit dem dazugehörigen Bedarf nach Restaurants und Kneipen betrachtete - insbesondere nach der erfolgten Renovierung des Platzes im Jahr 2001 - den Helmholtzplatz fortan als ihren Standort und ließ das entstehen, was mit einem gewissen Understatement als Nutzungskonflikt bezeichnet wird. Eine Lösung dieses Konfliktes erfolgte durch eine »ausgrenzende Einbeziehung« (Holm 2001: 8) unter Beteiligung des Quartiersmanagements, dass sich die Aufwertung des Gebietes zur Aufgabe und, gemeinsam mit Polizei und Bezirksamt, das Ziel der Verdrängung der genannten Randgruppen zu Eigen gemacht hat. Dazu wurde ein abgestuftes Konzept entwickelt: Während Benutzer illegal(isiert)er Drogen unter das Kuratel der Polizei gerieten - und diese auch gegen quartiersfremde Trinker vorging -, haben »Benutzer nicht illegaler Drogen, die in der Umgebung wohnen, [...] inzwischen fast so etwas wie eine Hausmeister- oder Platzwächterfunktion« gegenüber ortsfremden Trinkern übernommen und sind »damit selbst zum Teil der Kontroll- und Ausgrenzungsstrategien« geworden (Holm 2001: 9). Gegen ortsfremde Trinker, Punks und Trebekids wurde ergänzend durch das QM ein aus einer ABM finanzierter Nonprofit-Sicherheitsdienst installiert, der nun für das sorgen soll, was Quartiersmanagement und der neuen Bewohnerschaft als Sicherheit und Ordnung gilt.

19 Vgl. Holm (2001); Schulz zur Wiesch (2002: 89ff.); Eick (2003a: 374ff.). 
Das Kottbusser Tor im Bezirk Kreuzberg beherbergt die größte offene Drogenszene in Berlin und ist - wie das Quartiersmanagementgebiet Helmholtzplatz - zugleich ein >gefährlicher Ort` nach dem Berliner Allgemeinen Sicherheits- und Ordnungsgesetz (ASOG), an dem die jeweilige Polizeidirektion Bürgerrechte suspendieren, verdachtsunabhängige Taschen- und Identitätskontrollen anordnen und durchführen kann (Eick 2003b: 76). Es kann also keine Rede davon sein, dass sozialpolitische Problemlagen in QM-Gebieten ausschließlich sozial(integrativ) bearbeitet werden, denn eine ordnungs- und sicherheitspolitische >Aktivierung ist ebenso präsent. Doch auch unabhängig von polizeilichen Strategien wird im Quartiersmanagement >Ordnungspolitik ‘ betrieben. Das QMTeam am Kottbusser Tor beschreibt in Interviews die »erreichte >Verbesserung von Sauberkeit und Sicherheit als seinen größten Erfolg« (Fritsche 2003: 84, Hervorh. im Orig.) und trägt die Verantwortung dafür, dass die »bis dahin offenen Blockinnenhöfe im GSW-Bestand [die dortige städtische Wohnungsbaugesellschaft, ve] geschlossen« (ebd.) und so quasi zu QM-eigenen Gated Communities wurden. Das Quartiersmanagement richtete Concierge-Projekte ein, die auch regelmäßige Kontrollgänge durchführen, und »am Zentrum Kreuzberg nahm, unterstützt und teilweise auch finanziert durch das QM-Team, ein Sicherheitsdienst seine Arbeit auf « (ebd.). Fritsche betont, dass die MitarbeiterInnen des Quartiersmanagement aus ihrer QM-Sicht »auftragsgemäß auf den Vorschlag des Mieterbeirats« zur Einrichtung eines solchen Dienstes zu reagieren hatten (ebd.: 85), sich aber weder um die (Meinung der) 127 sonstigen Vereine im Quartier, noch um die Netzwerke zwischen den mehrheitlich türkischen Gewerbetreibenden kümmerten: "Sie werden als unerheblich, teilweise auch als nicht funktionsfähig eingestuft« (ebd.: 88f.).

Im QM-Gebiet Pankstraße im Wedding beschlossen im Jahr 2003 die dortige Kiez-AG und das Quartiersmanagement ebenfalls, gegen das dort ansässige Trinkermilieu vorzugehen und - »die Polizei sprach Platzverweise aus und kontrollierte verstärkt« (Schuster 2003: 12) - die dortigen Stadtmöbel sowie "sämtliche Hochbeete mit Pergolen und Bepflanzungen « zu entfernen: »Es gibt keine Rückzugsräume mehr für die Alkoholiker, sie werden sich dort nicht mehr wohl fühlen«, erklärt die Quartiersmanagerin; die zuständige Baustadträtin »befürwortet den Umbau« (ebd.). In vier weiteren QM-Gebieten Berlins arbeiten auf Antrag bzw. in Zusammenarbeit mit den Quartiersmanagements kommerzielle Sicherheitsdienste, in fünf Quartieren sind Sonderorganisationen der Polizei tätig, und in >Schwerpunktbezirken` ist das Ordnungsamt aktiv. 
Ein Blick in die Praxisdatenbank des Deutschen Institut für Urbanistik (DIfU), die insgesamt 433 Einträge ausweist, ${ }^{20}$ zeigt zudem, dass das Thema auch bundesweit von Relevanz im Quartiersmanagement ist: 52 Treffer für den Begriff >Sicherheit $<{ }^{21} 13$ für >Sauberkeit<, 45 für >Ordnung . Noch auf eine weitere Innovation im Quartiersmanagement muss hingewiesen werden: Aus einer Internet-Recherche nämlich geht hervor, dass in neun von seinerzeit (2004) 17 Berliner QM-Gebiete in Zusammenarbeit mit Beschäftigungsträgern lokale Sicherheits- und Ordnungsstreifen aufgebaut worden sind, die unter Begriffen wie ১Green Cops`, `Kiezläufer` und >Platzmeister` firmieren. Die genannten AlternativSicherheitsdienste, die keine hoheitlichen Rechte haben, werden heute vor allem aus Mitteln der Arbeitsagenturen finanziert. Sie sollen Hundehalter "zur Einhaltung des Leinenzwangs auffordern, Alkoholkonsumenten in den Grünanlagen auf Sauberkeit und Lärmvermeidung hinweisen [...], Spritzen einsammeln, durch Präsenz Vandalismus verhindern [...], gewalttätige Auseinandersetzungen versuchen zu schlichten, durch Präsenz Kriminalität verhindern « (Wauer 2002: 1f.). Die in diesen Alternativ-Polizeien Beschäftigten stammen zum Teil aus dem jeweiligen Quartier, in einigen Quartieren werden die Leistungen dieser Dienste evaluiert, deren Ergebnisse sind aber öffentlich nicht zugänglich.

\section{Lokales Ordnen im Wandel}

Es stellt sich die Frage, was das nun alles für die Ordnungen bzw., so mein Vorschlag, für das Ordnen im Wandel bedeutet. Eine Antwort kann zunächst mit Nikolas Rose (1996: 335) gegeben werden, wenn Quartiersmanagement als Regierungsform, als eine »Methode des Regierens« (Hervorh. im Original) betrachtet wird, die auf die Etablierung eigenverantwortlicher, sich selbst regierender Gemeinschaften zielt. Zahlreiche lokalstaatliche Programme der jüngeren Vergangenheit

"attempt to sempower the inhabitants of particular inner-city locales by constituting those who reside in a certain locality as sa community, by seeking out sommunity groups «ho can claim to speak sin the name of community and by linking them in new ways into the political apparatus in order to enact programmes which seek to regenerate the economic and human fabric of an area« (Rose 1996: 336, Hervorh. im Original).

20 Vgl. http://www.sozialestadt.de/praxisdatenbank/suche/ suchmaschine [09.11.2006].

21 Der Begriff /Sicherheitsgefühk erbringt zum selben Zeitpunkt 15 Treffer. 
Im Zusammenspiel mit einem neoliberalen Ethos, der individuelle Freiheit und Wahl (statt kollektiver Solidarität), Chancengerechtigkeit (statt Chancengleichheit), eigenverantwortliches Handeln, Eigeninitiative und Pflichterfüllung für die so genannten Benachteiligten in den Mittelpunkt der neuen lokalen Governance-Regime stellt, ist Quartiersmanagement so ein (letztes) Angebot an die BewohnerInnen sabgehängter Stadtquartiere, sich selber aus dem Sumpf (selbstverschuldeter) Armut und Abhängigkeit zu ziehen. Der Auftrag an professionelle Träger, die soziale Lage in den sbenachteiligten` Quartieren durch Empowerment der BewohnerInnen zu stabilisieren, eine ressort- und institutionenübergreifende Zusammenarbeit anzuregen sowie durch Interaktion den Prozess der Verwaltungsmodernisierung zu beschleunigen, kann daher als Versuch des (Lokal)Staats charakterisiert werden, eine "Zivilgesellschaft von oben« (Lanz 2000) zu etablieren. In dem Bemühen, - durchaus erkannte, aber systematisch de-thematisierte - neoliberale Verwüstungen auf Quartiersebene abzufedern, stellt Quartiersmanagement zugleich ein Instrument zur Durchsetzung und Absicherung eines lokalpolitisch flankierten Sozialstaatsumbaus á la Hartz dar. Während es sich beim BundLänder-Programm eher um ein paternalistisches Modellprogramm handelt, das - eingebunden in eine zunehmend punitive lokale Sozialstaatlichkeit - die Funktionsfähigkeit des restrukturierten Lokalstaats und den Gehorsam der QuartiersbewohnerInnen optimieren soll, ${ }^{22}$ gehen die neuen sozialstaatlichen Initiativen der beschäftigungspolitischen Aktivierung noch einen Schritt weiter. Klaus Ronneberger hat unlängst treffend auf den Punkt gebracht, dass aus der >Hilfe zur Selbsthilfe « der 1980er Jahre nun die (Wieder)Herstellung individueller Wettbewerbsfähigkeit als >Hilfe im Wettbewerb gide verknüpft mit dem Zwang zur unbedingten Arbeitsbereitschaft und lässt die alte Debatte über die würdigen und unwürdigen Armen wieder aufleben:

"Die neue Sozialpolitik schafft so die Situation, über berechtigte und unberechtigte Bedarfe urteilen zu müssen, was letztlich auf die altbekannte Unterscheidung vom swürdigen würdige erweist sich ökonomisch als >Investitionsruine〈, sozial als `Parasit«« (Ronneberger 2007, zit.n. Eick 2007a, Hervorh. im Original).

22 Um Missverständnissen vorzubeugen, ich sage nicht, es sei das ausschließliche Ziel des Quartiersmanagement, einen lokalen "Sicherheitsstaat« (Hirsch 1980) zu etablieren, es ist aber eines der - an Bedeutung gewinnenden - Ziele des Programms (vgl. Eick 2005). 
Für das lokale Ordnen im Wandel zeichnet sich mithin Folgendes ab: In der lokalen Sicherheitspolitik entsteht derzeit durch den Einsatz der Nonprofit-Sicherheitsdienste ein neuer, ordnungspolitisch agierender Akteur im öffentlichen Raum. Die Beschäftigung von Erwerbslosen und Sozialhilfeempfängern durch Nonprofits in Ordnungs- und Sicherheitprojekten leistet einen Beitrag zu einer neuen, hoch problematischen »culture of control" (Garland 2001). Die sich in einigen Quartieren herausbildenden Kontrollregime stellen dabei auch insoweit eine brisante Entwicklung dar, als hier Nonprofits in die lokale Sicherheitsproduktion eingebunden werden, ohne dass deren Legitimation geklärt oder entsprechende formale Qualifikationen nachgewiesen sind. Weiter beginnen sich unter Überschriften wie >Integration benachteiligter Gruppen< oder >Stabilisierung benachteiligter Quartiere` Strukturen herauszubilden, die auf ein "the poor policing the poor « hinauslaufen (Eick 2003a). Nonprofits agieren als Quasi-Polizeiagenturen, betreiben eine Containment-Politik gegen Armutspopulationen, weisen diesen damit spezifische Räume zu und vertreiben sie aus anderen.

\section{Literatur}

Alisch, M. (Hg.) 1998: Stadtteilmanagement. Voraussetzungen und Chancen für die soziale Stadt, Opladen: Leske + Budrich.

Anheier, H.K./E. Priller/W. Seibel/A. Zimmer (Hg.) 1999: Der Dritte Sektor in Deutschland. Organisationen zwischen Staat und Markt im gesellschaftlichen Wandel, Berlin: edition sigma.

Becker, H./R.-P. Löhr 2000: ')Soziale Stadt‘. Ein Programm gegen die sozialräumliche Spaltung in den Städten«. Aus Politik und Zeitgeschichte 10-11, S. 22-29.

Beste, H. 2001: "Zonale Raumkontrolle in Frankfurt/M. im ausgehenden 20. Jahrhundert«. In: M. Dinges/F. Sack (Hg.), Unsichere Großstädte? Vom Mittelalter bis zur Postmoderne, Konstanz: Universitätsverlag, S. 333-353.

Brand, U./W. Raza (Hg.) 2003: Fit für den Postfordismus? Gesellschaftspolitische Perspektiven des Regulationsansatzes, Münster: Westfälisches Dampfboot.

Breckner, I. 1997: »Europäische Modellvorhaben zur Armutsbekämpfung. Experimentierfelder zivilgesellschaftlichen Handelns in der Sozialpolitik?«. H. Heinelt/K.M. Schmals (Hg.), Zivile Gesellschaft. Entwicklungen, Potenziale und Defizite, Opladen: Leske + Budrich, S. 271-289.

Brenner, N./N. Theodore 2002: »Cities and the Geographies of >Actually Existing Neoliberalism «". Antipode 34, Nr. 3, S. 349-379.

Burney, E. 2005: Making People Behave. Anti-social Behaviour, Politics and Policy. Cullompton: Willan. 
Crawford, A./S. Lister, 2004: The Extended Policing Family. Visible Patrols in Residential Areas, Leeds: University of Leeds.

Dahme, H.-J./H.U. Otto/A. Trube/N. Wohlfahrt (Hg.) 2003: Soziale Arbeit für den aktivierenden Staat Opladen: Leske + Budrich.

DeFilippis, J./R. Fisher/E. Shragge 2006: »Neither Romance Nor Regulation: Reevaluating Community«. International Journal of Urban and Regional Research 30, Nr. 3, S. 673-689.

Demirovič, A./H.-P. Krebs/T. Sablowski (Hg.) 1992: Hegemonie und Staat. Kapitalistische Regulation als Projekt und Prozess, Münster: Westfälisches Dampfboot.

Dreher, G./T. Feltes (Hg.) 1997: Das Modell New York: Kriminalprävention durch »Zero Tolerance«? Beiträge zur aktuellen kriminalpolitischen Diskussion, Holzkirchen: Centaurus.

Eick, V. 2000: "Arbeit, Angst und Attraktionen. Arme gegen Arme und das Bernauer >Modell Bürgerhelfer «. Bürgerrechte \& Polizei/CILIP 66, Nr. 2, Berlin, S. 39-48.

Eick, V. 2003a: »New Strategies of policing the poor: Berlin's Neo-liberal Security System«. Policing \& Society 13, Nr. 4, S. 365-379.

Eick, V. 2003b: ")Und das ist auch gut so...< Polizieren im Berlin des 21. Jahrhunderts«. In: S. Nissen (Hg.), Kriminalität und Sicherheitspolitik. Analysen aus London, Paris, Berlin und New York, Opladen: Leske + Budrich, S. 6788.

Eick, V. 2004: »Kommando Bürgernähe. Die Kiezpolizei auf dem Weg in die Quartiere«. Berliner MieterEcho, 305, S. 14-15.

Eick, V. 2005: »Neoliberaler Truppenaufmarsch? Nonprofits als Sicherheitsdienste in >benachteiligten« Quartieren«. In: G. Glasze/R. Pütz/M. Rolfes (Hg.), Diskurs - Stadt - Kriminalität. Städtische (Un-)Sicherheiten aus der Perspektive von Stadtforschung und Kritischer Kriminalgeographie. Bielefeld: transcript, S. 167-202.

Eick, V. 2006: ")Contested Territory... $<$ Controlling Urban Spaces - New Actors in New Places«. Trialog. Zeitschrift für das Planen und Bauen in der Dritten Welt 89, Nr. 2, S. 4-8.

Eick, V. 2007a: Aktivieren und Strafen. Zur Kommunalisierung der sozialen Kontrolle unter Hartz IV (Vortrag auf dem Workshop der Stiftung W. »Einschluss $\mid$ Ausschluss | Umschluss. Die Hartz-Gesellschaft und ihre Kritik«, 3. März), Wuppertal.

Eick, V. 2007b: Neue Sicherheitskonzepte im sich wandelnden Wohlfahrtsstaat. Kommunale Kriminalpolitik zwischen Kommerzialisierung und Community, Berlin: i.E.

Eick, V./M. Mayer/B. Grell/J. Sambale 2004: Nonprofit-Organisationen und die Transformation der lokalen Beschäftigungspolitik, Münster: Westfälisches Dampfboot. 
Flint, J. (Hg.) 2006: R̊ousing, Urban Governance and Anti-social Behaviour. Perspertives, Folicy and Practice, Bristol: Policy Press.

Frashiz, T./R..P. Löhr 2000: »Überlegungen zum Quartiersmanagement«. Soziale Stacit 2, S. 2-4.

Fritsche, M. 2003: Neues Regieren im Quartier? Das Beispiel Kottbusser Tor in Berlin-Kreuzberg (FU Berlin, Otto-Suhr-Institut, unveröff. Diplomarbeit), Berlin.

Gangway e.V. (Hg.) 1997: Streetwork und Professionalität (Dokumentation einer Fachtagung zu Leistungsprofilen von Streetwork vom 15.-19. November 1996), Berlin: Selbstverlag.

Garland, D. 2001: The Culture of Control. Crime and Social Order in Contemporary Society, Oxford: Oxford University Press.

Gaston, K./J.A. Alexander 2001: "Effective Organisation and Management of Public Sector Volunteer Workers: Police Special Constables«. The International Journal of Public Sector Management 14, Nr. 1, S. 59-74.

Gramsci, A. 1991ff: Gefängnishefte. Kritische Gesamtausgabe, Hamburg/Berlin: Argument.

Grimm, G. 2004: Stadtteilentwicklung und Quartiermanagement. Entwicklung und Aufbau lokalspezifischer Organisations- und Steuerungsstrukturen, Essen: Klartext.

Häußermann, H./A. Kapphan 2000: Berlin: Von der geteilten zur gespaltenen Stadt? Sozialräumlicher Wandel seit 1990, Opladen: Leske + Budrich.

Harvey, D. 2005: A Brief History of Neoliberalism, Oxford: Oxford University Press.

Helms, G. 2003: Towards Safe City Centres? Remaking the Spaces of an Oldindustrial City (Dissertation), Glasgow: University of Glasgow.

Hinte, W. 1998: "Bewohner ermutigen, aktivieren, organisieren - Methoden und Strukturen für ein effektives Stadtteilmanagement«. In: M. Alisch (Hg.), Stadtteilmanagement. Opladen, S. 153-170.

Hirsch, J. 1980: Der Sicherheitsstaat. Das >Modell Deutschland<, seine Krise und die neuen sozialen Bewegungen, Frankfurt/M.: Europäische Verlagsanstalt.

Hirsch, J. 2002: Herrschaft, Hegemonie und politische Alternativen, Hamburg: VSA.

Hitzler, R. 1994: »Die neuen Vigilanten. Über Formen der Bewältigung alltäglicher Verunsicherung«. Bürgerrechte \& Polizei/CILIP 48, Nr. 2, S. 67-71.

Holm, A. 2001: "Ausgrenzende Einbeziehung - Flexible Kontrollstrategien am Helmholtzplatz«. Berliner MieterEcho 288, S. 8-10.

Hohmeyer, C. 2000: »Wachsame Nachbarn. Lokale Gemeinschaften im Dienst der Sicherheit«. Bürgerrechte \& Polizei/CILIP 66, Nr. 2, S. 51-58.

Jessop, B. 2002: "Liberalism, Neoliberalism, and Urban Governance: A Statetheoretical Perspective«. In: N. Brenner/N. Theodore (Hg.), Spaces of Neoliberalism, Oxford: Blackwell, S. 105-125. 
Johnston, L. 2005: »From `Community< to `Neighbourhood< Policing: Police Community Support Officers and the >Police Extended Family in London«. Journal of Community \& Applied Social Psychology 15, Nr. 3, S. 241-254.

Kebir, S. 1991: Gramsci's Zivilgesellschaft. Alltag, Ökonomie, Kultur, Politik, Hamburg: VSA.

Kessl, F./H.-U. Otto (Hg.) 2003: Soziale Arbeit und Soziales Kapital. Zur Kritik lokaler Gemeinschaftlichkeit, Opladen: VS Verlag für Sozialwissenschaft.

Kürpick, S./M. Murböck 1997: Kriminalprävention in Stadtteilen mit besonderem Erneuerungsbedarf (ILS-Schriften, Bd. 124), Dortmund: ILS.

Kury, H. (Hg.) 1997: Konzepte Kommunaler Kriminalprävention. Sammelband der $>$ Erfurter Tagung<, Freiburg/Brsg.: edition iuscrim.

Lanz, S. 2000: »Der Staat verordnet die Zivilgesellschaft«. Widersprüche 20, Heft 78, S. 39-51.

Leo, M. 2006: »Regierung will Hartz-IV-Empfänger in Bussen und Bahnen patrouillieren lassen«. Berliner Zeitung vom 28. August 2006, S. 1.

Levitas, R. 1998/2005: The Inclusive Society? Social Exclusion and New Labour. New York: Palgrave Macmillan.

Lindenberg, M. 2000: "Zwischen sicher sein und sich sicher fühlen. Kommunale Hilfsdienste als Geburtshelfer für verdichtete städtische Gemeinschaften?«. Widersprüche 20, Heft 76, S. 37-49.

Mahlberg, L. 1988: Gefahrenabwehr durch gewerbliche Sicherheitsunternehmen. Westberlin: Duncker \& Humblot.

Mayer, M. 2003: "The Onward Sweep of Social Capital: Causes and Consequences for Understanding Cities, Communities and Urban Movements«. International Journal of Urban and Regional Research 27, Nr. 1, S. 110-132.

McArdle, A./T. Erzen (Hg.) 2001: Zero Tolerance. Quality of Life and the New Police Brutality in New York City. New York: New York University Press.

Nitz, G. 2000: Private und öffentliche Sicherheit. Berlin: Duncker \& Humblot.

Nogala, D. 1995: »Was ist eigentlich so privat an der Privatisierung sozialer Kontrolle?«. In: F. Sack/M. Voß et al. (Hg.), Privatisierung staatlicher Kontrolle: Befunde, Konzepte, Tendenzen, Baden-Baden: Nomos, S. 234-260.

Olschok, H. 2004: "Entwicklung und Perspektiven des Wach- und Sicherheitsgewerbes auf nationaler und europäischer Ebene«. In: R. Stober/H. Olschok (Hg.), Handbuch des Sicherheitsgewerberechts, München: C.H. Beck, S. 1334.

Oschmann, F. 2005: Die Finanzierung der Inneren Sicherheit am Beispiel von Polizei und Sicherheitsgewerbe, Köln: Heymanns.

Peck, J./A. Tickell 2002: »Neoliberalizing Space«. Antipode 34, Nr. 3, S. 380404.

Peck, J./A. Tickell 2007: Conceptualizing Neoliberalism, Thinking Thatcherism«. In: H. Leitner/J. Peck/E. Sheppard (Hg.), Contesting Neoliberalism. Urban Frontiers, New York: Guilford Press, S. 26-50. 
Pitschas, R. 2000: Polizei und Sicherheitsgewerbe. Rechtsgutachten zu verfassungs- und verwaltungsrechtlichen Aspekten der Aufgabenverteilung zwischen Polizei und privaten Sicherheitsunternehmen, Wiesbaden: Bundeskriminalamt.

Pütter, N. 2002: »Kommunalpolitik als Kriminalpolitik. Über die Verwandlung des Politischen in der Präventionsgesellschaft«. In: R. Prätorius (Hg.), Wachsam und kooperativ? Der lokale Staat als Sicherheitsproduzent, Baden-Baden: Nomos, S. 64-79.

Pütter, N. 2006: Polizei und kommunale Kriminalprävention. Formen und Folgen polizeilicher Präventionsarbeit in den Gemeinden, Frankfurt/M.: Verlag für Polizeiwissenschaft.

Rigakos, G. 2002: The New Parapolice. Risk Markets and Commodified Social Control, Toronto: University of Toronto Press.

Robertson, R. 1998: »Globalisierung: Homogenität und Heterogenität in Raum und Zeit«. In: U. Beck (Hg.), Perspektiven der Weltgesellschaft, Frankfurt/M.: Suhrkamp.

Rose, N. 1996: "The Death of the Social? Re-figuring the Territory of Government«. Economy and Society 5, Nr. 3, S. 327-256.

Schuleri-Hartje, U.-K. 2002: "Vorbeugen ist besser als Bestrafen. Das Programm 'Soziale Stadt holt alle mit ins Boot«. der städtetag 11, S. 23-26.

Schulz zur Wiesch, L. 2002: Ein Platz für Alle. Quartiersmanagement am Helmholtz-Platz in Berlin-Prenzlauer Berg (HU Berlin, Institut für Sozialwissenschaften, unveröff. Diplomarbeit), Berlin: Humboldt Universität zu Berlin.

Schuster, S. 2003: »Utrechter Platz. Aufwerten durch Abräumen«. Berliner MieterMagazin 6, S. 12-14.

Senatsverwaltung für Stadtentwicklung Berlin (Hg.) 1998: Sozialorientierte Stadtentwicklung (Gutachten), Berlin: Selbstverlag.

Spindler, H. 2003: ")Überfordern und überwachen`. Der restriktive Paradigmenwechsel in der Sozialpolitik«. Sozial extra 8, Nr. 9, S. 11-14.

Stober, R./H. Olschok (Hg.) 2004: Handbuch des Sicherheitsgewerberechts, München: C.H. Beck.

Swyngedouw, E. 1997: »Neither Global nor Local: >Glocalisation< and the Politics of Scale«. In: K. Cox (Hg.), Spaces of Globalization: Reasserting the Power of the Local, New York: Guilford Press, S. 137-166.

Trotha, T. v. 2005: »Der Aufstieg des Lokalen«. Aus Politik und Zeitgeschichte 28-29, S. 32-38.

Walther, U.-J. (Hg.) 2002: Soziale Stadt - Zwischenbilanzen. Ein Programm auf dem Weg zur Sozialen Stadt? Opladen: Leske + Budrich.

Wauer, B. 2002: Kiezläufer für das Quartier Reinickendorfer/Pankstraße, http://www.pankstrasse-quartier.de/156.html. 